\title{
$n+{ }^{235} \mathrm{U}$ resonance parameters and neutron multiplicities in the energy region below $100 \mathrm{eV}$
}

\author{
Marco T. Pigni ${ }^{1, a}$, Roberto Capote ${ }^{2}$, Andrej Trkov², and Vladimir G. Pronyaev ${ }^{3}$ \\ 1 Oak Ridge National Laboratory, P.O. Box 2008, Oak Ridge, TN, USA \\ 2 International Atomic Energy Agency, Nuclear Data Section, Austria \\ 3 State Corporation Rosatom, Moscow, Russian Federation
}

\begin{abstract}
In August 2016, following the recent effort within the Collaborative International Evaluated Library Organization (CIELO) pilot project to improve the neutron cross sections of ${ }^{235} \mathrm{U}$, Oak Ridge National Laboratory (ORNL) collaborated with the International Atomic Energy Agency (IAEA) to release a resonance parameter evaluation. This evaluation restores the performance of the evaluated cross sections for the thermaland above-thermal-solution benchmarks on the basis of newly evaluated thermal neutron constants (TNCs) and thermal prompt fission neutron spectra (PFNS). Performed with support from the US Nuclear Criticality Safety Program (NCSP) in an effort to provide the highest fidelity general purpose nuclear database for nuclear criticality applications, the resonance parameter evaluation was submitted as an ENDF-compatible file to be part of the next release of the ENDF/B-VIII.0 nuclear data library. The resonance parameter evaluation methodology used the Reich-Moore approximation of the $R$-matrix formalism implemented in the code SAMMY to fit the available time-of-flight (TOF) measured data for the thermal induced cross section of $\mathrm{n}+{ }^{235} \mathrm{U}$ up to $100 \mathrm{eV}$. While maintaining reasonably good agreement with the experimental data, the validation analysis focused on restoring the benchmark performance for ${ }^{235} \mathrm{U}$ solutions by combining changes to the resonance parameters and to the prompt resonance $\bar{v}$ below $100 \mathrm{eV}$.
\end{abstract}

\section{Introduction}

Evaluated on the basis of direct measurements in the neutron thermal energy range, a new set of TNCs [1] features a reduction of the neutron thermal multiplicity $\bar{v}$ and an increase of the fission cross section at thermal energy. Moreover, a new evaluation of the PFNS [2] in the same energy range has determined a lower value of the average neutron energy than that reported in the existing evaluated nuclear data libraries. Simulations on a number of thermal-solution benchmarks has shown that the combined use of the new TNCs and a softer prompt fission neutron spectrum at thermal energy yields $k_{\text {eff }}$ values that are larger than measurements by a margin that increases as the above-thermal-leakage fraction (ATLF) increases. Although considered an important constraint for the evaluated physical quantities, the performance between $k_{\text {eff }}$ values in well-thermalized and above-thermal systems

\footnotetext{
a e-mail: pignimt@ornl.gov Notice: This manuscript has been authored by UT-Battelle, LLC under Contract No. DE-AC0500OR22725 with the U.S. Department of Energy. The United States Government retains and the publisher, by accepting the article for publication, acknowledges that the United States Government retains a non-exclusive, paid-up, irrevocable, worldwide license to publish or reproduce the published form of this manuscript, or allow others to do so, for United States Government purposes. The Department of Energy will provide public access to these results of federally sponsored research in accordance with the DOE Public Access Plan (http:// energy.gov/downloads/doe-public-access-plan).
}

for ${ }^{235} \mathrm{U}$ solutions was achieved almost independently in the current nuclear data libraries.

Although conceived as an intermediate step in the evaluation of the ${ }^{235} \mathrm{U}$ resonance parameters and, currently, included in the $\beta_{2}$ release of the ENDF/ B-VIII.0 nuclear data library [3], this work also restored the benchmark performance of the ${ }^{235} \mathrm{U}$ solutions by combining changes to the resonance parameters, with changes to the prompt resonance $\bar{v}$ below $100 \mathrm{eV}$. This new set of physical quantities restored the agreement for high-leakage-solution benchmarks while maintaining the good performance of large thermal solution assemblies. Additionally, the new set of resonance parameters yields cross sections in reasonable agreement with the available TOF-measured data for neutron-induced fission of ${ }^{235} \mathrm{U}$ from thermal up to $100 \mathrm{eV}$.

The computer code SAMMY [4] was used to analyze experimental data in the resolved resonance region (RRR) up to $100 \mathrm{eV}$ using the multilevel multi channel $R$-matrix representation of the neutron cross sections defined according to the Reich-Moore (RM) approximation. Experimental conditions such as resolution function, finite size sample, nonuniform thickness, and nuclide abundances of sample, multiple scattering, self-shielding, normalization, background, and Doppler broadening were taken into account in performing the analysis. Though the current analysis on the resonance parameters was limited to $100 \mathrm{eV}$, the entire evaluation extends to neutron incident energies up to $2.25 \mathrm{keV}$, and the $s$-wave resonances have spin and parity, $3^{-}$and $4^{-}$, as displayed in Table 1. 
Table 1. Neutron energy range for the ${ }^{235} \mathrm{U}$ evaluation and the number of fitted levels for the two channel spins related to $s$-wave neutrons.

\begin{tabular}{lccrl}
\hline Nucleus $\left(I^{\pi}\right)$ & $E_{\max }(\mathrm{keV})$ & Method & $J_{3-}$ & $J_{4-}$ \\
\hline${ }^{235} \mathrm{U}\left(7 / 2^{-}\right)$ & 2.25 & $\mathrm{RM}$ & 1433 & 1731 \\
\hline
\end{tabular}

To achieve the integral fission value suggested by recently measured fission TOF data [2], emphasis on producing the current resonance evaluation was devoted not only to the sub thermal and thermal regions by including thermal constants based on microscopic data, but also to neutron incident energies between $7.8-11 \mathrm{eV}$. Moreover, the newly evaluated neutron multiplicities included fluctuations (Sect. 2). And, among the set of experimental data, the Brooks' measurements [5] on $\alpha=\sigma_{\gamma} / \sigma_{\mathrm{f}}$ or, equivalently, $\eta=v \cdot(1+\alpha)^{-1}$ (being $v$ a normalization factor related to the average number of neutron per fission) in the neutron incident energy range up to $20 \mathrm{eV}$ (Sect. 3) were included in the analysis. The results of the benchmark simulations for thermal solutions as a function of the ATLF are presented and briefly discussed in Sect. 3, followed by conclusions in Sect. 4.

\section{Neutron multiplicities}

The quantity $\bar{v}$ represents the average value of the prompt neutron emission multiplicity distribution $P_{v}$ and correlates each fission event occurring in a nucleus with the emission of prompt neutrons. High-resolution measurements on the average number of prompt neutrons $\bar{v}$ depending on the incident neutron energy has been performed for the most important fissile materials. An example of these measured data for neutrons incident on ${ }^{235} \mathrm{U}$ target is given in Fig. 1. Here, several data sets from different authors [6-10] are plotted normalized to the $\bar{v}\left(E_{\text {th }}\right)$ calculated at the thermal energy $(0.0253 \mathrm{eV})$. In the observed average number of neutrons there are clear fluctuations that can be roughly correlated with the energy of compound nucleus resonances, but also partially explained by the influence of the $(n, \gamma f)$ process, where a $\gamma$ is emitted prior to the fission event. In this process the excitation energy available for the fission reaction channel is diminished and this leads to a lower neutron multiplicity. In JEFF-2.2 nuclear data library (black solid line) these fluctuations were included in the evaluated data, however, in all other modern libraries (including ENDF/B-VII.1 library plotted in red solid line) they have been neglected. The current evaluation in the ENDF/B-VIII. $0 \beta_{2}$ release restored the fluctuating trend-line of the average number of prompt neutrons as displayed by the blue solid line in Fig. 1.

\section{Cross section and benchmark results}

The ${ }^{235} \mathrm{U}$ resolved resonance evaluation recently released within the ENDF/B-VIII.0 $\beta_{2}$ nuclear data library [3] was developed on the basis of newly evaluated thermal neutron constants [1], as well as on new PFNS [2]. In the thermal energy range, this work relies on the values of the fission and capture cross sections reported in Table 2, along with the values of the evaluated cross sections reconstructed for $\mathrm{T}=0 \mathrm{~K}$.

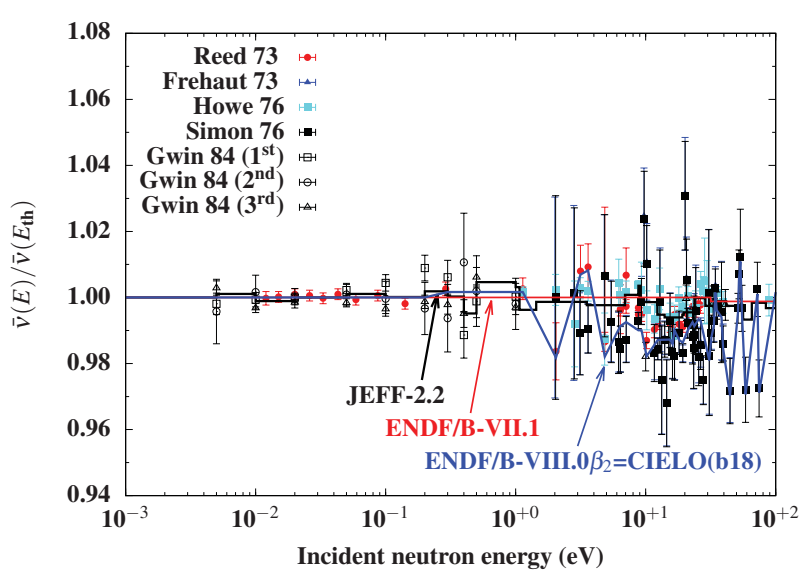

Figure 1. $\bar{v}(E)$ relative to $\bar{v}\left(E_{\mathrm{th}}\right)$ calculated at thermal energy $(0.0253 \mathrm{eV})$ for $\mathrm{n}+{ }^{235} \mathrm{U}$ reactions. Several experimental data sets are compared to three evaluated data.

Table 2. Thermal cross sections in barn compared to the neutron standards and CONRAD values [1]. Derived quantities like $\alpha$ and $\eta$ (computed for $v=1$ ) are also reported.

\begin{tabular}{cccc}
\hline ORNL $^{\mathrm{a}}$ & Standards & CONRAD & Quantity \\
\hline 586.6 & $587.15 \pm 1.36$ & $586.4 \pm 1.5$ & $\sigma_{\mathrm{f}}$ \\
99.4 & $99.26 \pm 1.36$ & $99.1 \pm 2.1$ & $\sigma_{\gamma}$ \\
14.08 & $14.09 \pm 0.21$ & $14.05 \pm 0.22$ & $\sigma_{\mathrm{el}}$ \\
0.855 & 0.855 & 0.855 & $\eta^{\mathrm{b}}$ \\
0.1694 & 0.1690 & 0.1689 & $\alpha^{\mathrm{b}}$ \\
\hline
\end{tabular}

${ }^{\mathrm{a}} \mathrm{T}=0 \mathrm{~K}$.

${ }^{\mathrm{b}}$ Calculated from $\sigma_{\mathrm{f}}$ and $\sigma \gamma$.

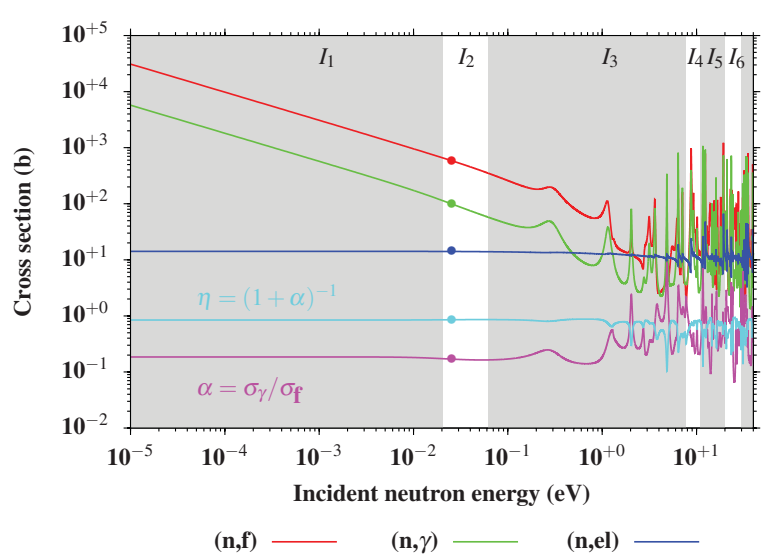

Figure 2. $n+{ }^{235} \mathrm{U}$ reconstructed cross sections from the ORNL resonance parameter evaluation in thermal energy range up to $40 \mathrm{eV}$. For $\eta$ the quantity $v$ was considered unity.

Figure 2 shows the reconstructed cross sections for the fission, capture, and elastic reaction channels. Derived quantities such as $\alpha$ and $\eta$ (for which, in the plot, $v=1$ ) are also displayed. In the evaluation analysis, constraints on the fission and capture integrals in selected energy regions $\left(I_{1}, \ldots, I_{6}\right)$ were satisfied to reach reasonable agreement with both differential and integral data. In particular for differential data, $I_{4}$ refers to the integral in the neutron energy range between $7.8-11 \mathrm{eV}$ whose standard reference value is $246.4 \mathrm{~b} \cdot \mathrm{eV}$.

For energies below $100 \mathrm{eV}$, this work restores benchmark performance for ${ }^{235} \mathrm{U}$ solutions by combining changes to the prompt resonance $\bar{v}$ and the resonance parameters. In achieving this, the present set of resonance 


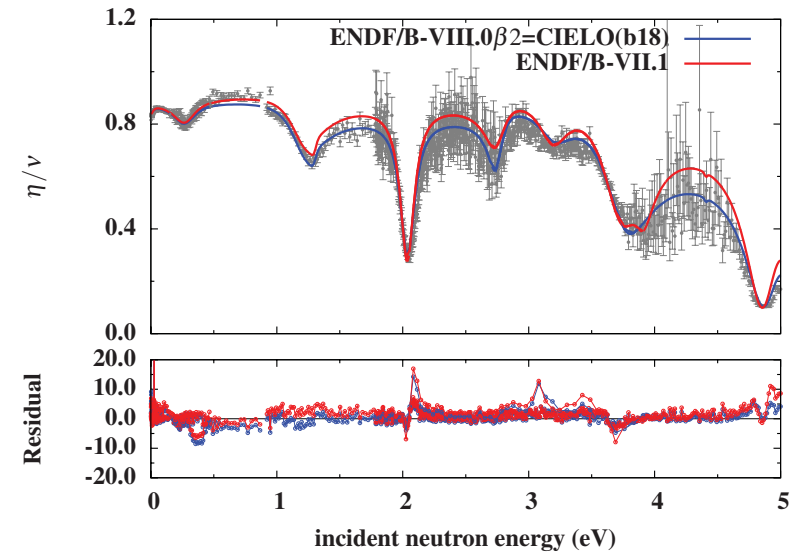

Figure 3. $\mathrm{n}+{ }^{235} \mathrm{U} \eta$ measurements of Brooks [5], Wartena and Weigmann [11] compared to ENDF/B-VII.1 [12] and ENDF/BVIII. $0 \beta_{2}$ values.

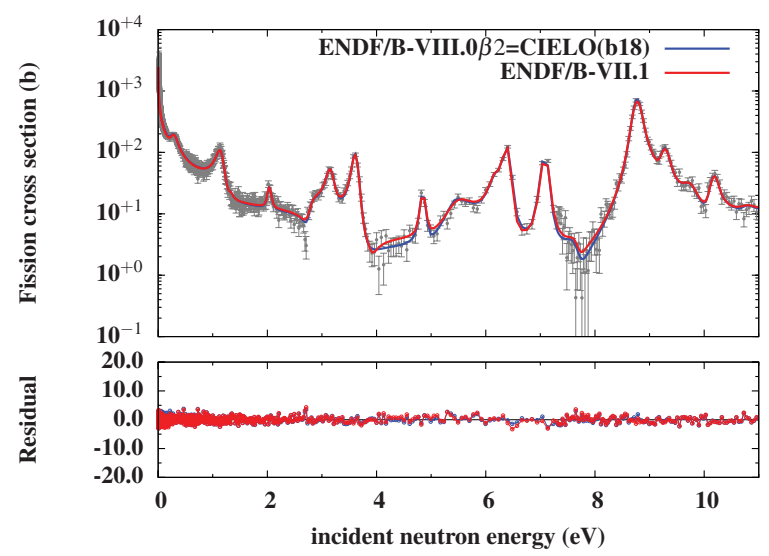

Figure 4. $\mathrm{n}+{ }^{235} \mathrm{U}$ Gwin's fission measurement compared to ENDF/B-VII.1 [12] and ENDF/B-VIII.0 $\beta_{2}$ values.

parameters yields cross sections still in reasonable agreement with the suite of experimental data included in the previous resonance evaluations. Additionally, the set of $\eta$ measurements performed by Brooks [5] in the mid-1960s at the Atomic Energy Research Establishment (Harwell) were analyzed and included in the fit for incident neutron energies up to $20 \mathrm{eV}$. Figure 3 displays multiple measurements of Brooks [5] in the incident neutron energy range up to $5 \mathrm{eV}$ and also measured data of Wartena and Weigmann [11] in the low-energy range up to $0.5 \mathrm{eV}$.

All measurements are normalized to the reported $v$ value. The comparison of ENDF/B-VII.1 (in red) and ENDF/B-VIII. $0 \beta_{2}$ (in blue) $\eta$ values is also shown. Although there are large uncertainties above $2 \mathrm{eV}$, the CIELO $\eta$ (decreased) values are on average in better agreement with the experimental data than ENDF/BVII.1 values. This was achieved by increasing the capture cross sections mostly in the valley of the resonances while keeping their peak values unchanged. The resonance at $E_{\mathrm{n}}=2 \mathrm{eV}$ is clearly an example. The sensitivity of the resonance parameters to fission cross sections seems to be more relevant than to capture cross sections at neutron energies $\gtrsim 4 \mathrm{eV}$. This effect is evident in the fission cross sections shown in the blue continuous line in Fig. 4 in which Gwin's fission cross section measurements are displayed along with ENDF/B-VII.1 and ENDF/ B-VIII. $0 \beta_{2}$ values.

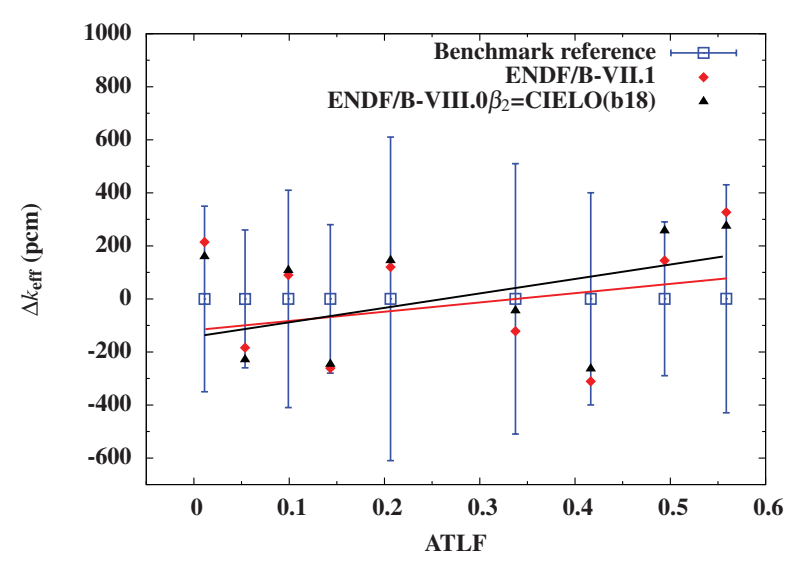

Figure 5. Differences (in pcm) between predicted and measured $k_{\text {eff }}$ of ATLF benchmarks using ENDF/B-VII.0 (in red) and the ENDF/B-VIII. $0 \beta_{2}$ evaluation (in black).

The decreased neutron production suggested by Brooks' data also seemed consistent with the use of a softer PFNS and the newly fitted thermal neutron constants in order to compensate the decreased criticality. Moreover, the values of the resonance parameters were constrained by cross section integrals, e.g., the fission integral in the incident energy range between $7.8-11 \mathrm{eV}$,

$$
I_{\mathrm{f}}=\int_{E=7.8 \mathrm{eV}}^{E=11 \mathrm{eV}} \sigma_{\mathrm{f}}(E) d E=247.0 \mathrm{~b} \cdot \mathrm{eV}(\text { current value }),
$$

recommended early by the Wagemans [13] and later by Carlson [14] in the international evaluation of neutron cross section standards. Currently, this value is adopted as a normalization factor by the newly measured TOF cross section data [15].

In Fig. 5 the results of the suite of integral benchmarks for thermal solutions of highly enriched uranium (HEUSOL-THERM) are displayed as a function of the ATLF. Integral benchmarks were not used in the fitting procedure, but they provided a guideline to identify weaknesses in the evaluation. This resulted in evaluated resonance parameters that show a red solid trend line comparable to the ENDF/B-VII.1 black trend line with minimal deviation from the measured values, well within the experimental uncertainties.

\section{Conclusion and outlook}

Based on the recent effort to improve the $n+{ }^{235} U$ cross sections in the energy region below $100 \mathrm{eV}$, the $R$-matrix SAMMY method was used with the RM approximation to determine a consistent, but preliminary, set of resonance parameters. In the analyzed energy range, this set of resonance parameters restores the performance of the evaluated cross sections for the thermal- and abovethermal-solution benchmarks on the basis of newly evaluated TNCs and PFNS. At the same time, reasonable agreement with the experimental data is preserved. This work was motivated by the need to generate nuclear evaluated data that perform well in transport simulations and are exempt from any compensating errors. This reflects the goal of the CIELO collaboration: to provide a framework for nuclear data evaluation to establish the highest fidelity general purpose nuclear database. 


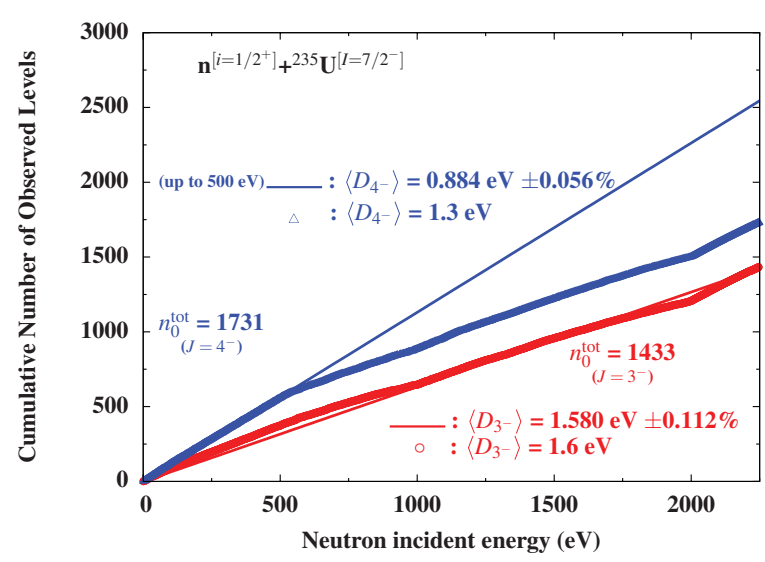

Figure 6. Plot of the cumulative number of observed $s$-levels (in blue triangles and red dots) vs energy for $n+{ }^{235} \mathrm{U}$ in the ENDF/B-VII.1 nuclear data library. The values of average $s$-level spacings for two $J$-spin populations $\left\langle D_{\ell}^{J}\right\rangle$ obtained by the fit of the observed levels (solid blue and red lines) are shown in the plot.

Future efforts include improving the evaluation work by including newly measured data for fission and capture cross sections, namely from the RPI and nTOF collaborations, to evaluate the cross sections in a more comprehensive energy range. This would allow for a complete and energetically comprehensive suite of validation tests to be conducted within the ENDF/B-VIII.0 nuclear data library. In particular, future work will include an improved set of resonance parameters for incident neutron energies above $500 \mathrm{eV}$. For these energies, the present evaluation is based on the ENDF/B-VII.1 nuclear data library $[12,16]$, in which the set of resonances were considered to be pseudo-resonances with different statistical properties than the true resonances in the energy range up $100 \mathrm{eV}$. This means a significant change in the observed level density, particularly for resonances of channel spin $J=4^{-}$(blue triangles) as illustrated in Fig. 6 .

An immediate consequence of correcting the statistical behavior of the level for energies above $500 \mathrm{eV}$ will be a new analysis of the external levels.
Another important inclusion will be the improvement of the distribution of the fission widths. Analyses of the ${ }^{235} \mathrm{U}$ fission widths [17] showed that the number of degrees of freedom does not obey the chi-squared distribution. This arises from the behavior of fission widths that fluctuate strongly from resonance to resonance, resulting in an unequal average of the total fission widths.

This work was supported by the US Department of Energy (DOE), Nuclear Criticality Safety Program (NCSP) funded and managed by the National Nuclear Security Administration for DOE.

\section{References}

[1] V.G. Pronyaev et al., ND2016 Conference Proceedings (to be published)

[2] R. Capote et al., Nuclear Data Sheets 131, 1 (2016)

[3] D.A. Brown, ND2016 Conference Proceedings (to be published)

[4] N.M. Larson, ORNL/TM-9179/R8 (2008)

[5] F.D. Brooks et al., AERE-M1670

[6] R.L. Reed et al., Chicago Operations Office 29, 3 (1972)

[7] J. Frehaut et al., Phys. Lett. B 42, 344 (1972)

[8] R.E. Howe et al., Phys. Rev. 13, 195 (1976)

[9] G. Simon et al., All Union Conf. on Neutron Phys. 5, 337 (1975)

[10] R. Gwin et al., Nucl. Sci. Eng. 87, 381 (1984)

[11] H. Weigmann et al., Int. Conf. on the Physics of Reactors 3, 33 (1990)

[12] M.B. Chadwick et al., Nuclear Data Sheets 112, 2887 (2011)

[13] C. Wagemans et al., IAEA/TECDOC-335 155 (1984)

[14] A.D. Carlson et al., International Evaluation of Neutron Cross-section Standards (2007)

[15] C. Paradela et al., EPJ Web of Conf. 111, 02003 (2016)

[16] L.C. Leal et al., Nucl. Sci. Eng. 131, 230 (1999)

[17] C.E. Porter et al., Phys. Rev. 104, 483 (1956) 